\title{
À propos d'une récente histoire européenne de la traduction
}

\section{YVES CHEVREL}

DOI : https://doi.org/10.31577/WLS.2021.13.3.12

"Histoire européenne de la traduction " : un titre prometteur ! Les auteurs d'Europäische Übersetzungsgeschichte, Jörn Albrecht et Iris Plack, le savent bien : aussi avertissent-ils d'emblée qu'ils se gardent de toute prétention d'exhaustivité ou de représentativité, et qu'ils proposent, en fait, un « choix de quelques faits et aspects systématiques de l'activité traductive en Europe de l'Antiquité à nos jours » (Albrecht et Plack 2018, 15). Le sommaire de leur ouvrage de 548 pages n'en est pas moins impressionnant : organisé en deux grandes parties (I : historique ; II : systématique), s'achevant par une Bibliographie et un Index commodes, il offre, sur la base de travaux récents, une mise en évidence de repères essentiels dans une enquête qui porte, pour l'essentiel, sur les traductions réalisées en Europe occidentale et en Russie. Cet ouvrage renouvelle largement le travail de pionnier d'Henri Van Hoof Histoire de la traduction en Occident (1991), qui n’avait pas été remplacé.

La partie historique rappelle qu'en Europe les débuts de la littérature latine sont liés aux traductions et aux adaptations : c'est l'occasion d'une mise au point sur des interprétations, parfois fallacieuses, de textes célèbres de Cicéron, Horace et Saint Jérôme. Suit, au Moyen Âge, la montée en puissance des langues vernaculaires, qui s'enrichissent grâce aux traductions, lesquelles permettent aussi à des genres littéraires de passer dans de nouveaux domaines linguistiques. Ces premiers chapitres (2 à 4), qui forment un total d'une cinquantaine de pages, sont le prélude à une série d'autres (5 à 8), plus spécifiques, d'une soixantaine de pages, portant sur la complexité (Vielschichtigkeit) de la formation de la langue anglaise, la traduction de la Bible, les « belles infidèles » et leur persistance au-delà des XVII e et XVIII ${ }^{e}$ siècles, l'importance du tournant de l'époque romantique, particulièrement sensible en Allemagne. Le point d'aboutissement est le chapitre 9, consacré à " L'histoire de la traduction d'œuvres choisies de la littérature universelle (Die Übersetzungsgeschichte ausgewählter Werke der Weltriteratur) ». Ce chapitre est le cœur de la partie historique, tant par la quantité des informations qu'il contient et la qualité des commentaires que par son organisation. En effet six espaces linguistiques sont présentés dans l'ordre «chronologique » de leur accession (Aufstieg) successive au rang - culturel - de langues de traduction; d'autre part, chacun d'eux est traité dans une double perspective d'intraduction 
et d'extraduction (les auteurs emploient les termes forgés en français pour désigner les œuvres traduites d'autres langues et les œuvres traduites en d'autres langues). Défilent ainsi, dans l'ordre (sous-chapitres 9.1 à 9.6) : l'italien, fondé sur le dialecte toscan, avec les traductions rapides des tre corone : Pétrarque, Boccace, Dante ; puis les langues de «l'Espagne et [de] la péninsule ibérique », quasi-exclusivement le castillan (le portugais étant traité un peu plus loin), le français, langlais (Grande Bretagne et Irlande), l'allemand (incluant l'Autriche et la Suisse), et enfin le russe. Le sous-chapitre 9.7 regroupe quelques " autres pays européens » : Portugal, Belgique, pays scandinaves, Pologne. Un dernier sous-chapitre (9.8), consacré aux « Métamorphoses du panthéon », donne aux auteurs l'occasion d'esquisser une typologie des possibles mutations de statut (Statusverschiebungen) opérées par la réception d'une traduction dans une communauté linguistique ; ils distinguent trois cas : 1) une œuvre, connue et appréciée de longue date, acquiert finalement une forme canonique traduite qui la place à l'égal des œuvres de la langue de cette communauté (La Bible de Luther, la King James Version) ; 2) une traduction nouvelle d'une œuvre déjà connue incite à des interprétations nouvelles qui peuvent, en retour, exercer une influence sur la culture-source (traduction du Quijote par Ludwig Tieck, qui modifie profondément les lectures de cette œuvre en Europe) : ils y voient une «métamorphose», en un sens restreint ; 3) une traduction permet de découvrir une œuvre jusqu'alors inconnue dans une communauté linguistique, ce qui peut entraîner une réévaluation des auteurs (ou des écrivains) déjà connus : romanciers russes de la fin du XIX ${ }^{e}$ siècle. Cette dernière situation, qu'ils appellent " métamorphose au sens large ", est particulièrement intéressante, et serait d'ailleurs à élargir : les auteurs " canoniques " propres à la culture réceptrice peuvent être aussi concernés par une réévaluation, comme dans le cas de la découverte, assez tardive, des romantiques allemands par les lecteurs français. La partie historique se conclut par un chapitre consacré à dix personnalités traduisant vers l'allemand (à l'exception d'Henri Albert, promoteur de Nietzsche en France) ; on note que cette série de portraits s'ouvre et se ferme sur deux traductrices : Élisabeth de Lorraine, comtesse de Nassau-Sarrebruck, qui traduisit ou fit traduire, au $\mathrm{XV}^{\mathrm{e}}$ siècle, quatre romans de gestes français, et la "dame aux cinq éléphants ", Svetlana Geier (1923-2010), connue pour ses traductions en allemand des cinq grands romans de Dostoïevski, mais aussi de nombreuses œuvres russes.

La seconde grande partie "systématique " comprend six chapitres (11 à 16). Le premier est consacré à la "balance commerciale des traductions en Europe » : les auteurs se déclarent conscients de la nouveauté (relative) d'un tel vocabulaire, qui leur semble devoir exiger une certaine accoutumance (gewöhnungsbedürftig); beaucoup d'éléments économiques interviennent de fait dans le marché des traductions, mais aussi des traditions relatives à la conception plus ou moins étroite de la « haute » littérature ou, par exemple, de la place reconnue à la littérature pour la jeunesse. Interviennent aussi les tentatives de contrôle (censures, saisies) exercées par le pouvoir politique, et les multiples façons de les contourner : les pseudo-traductions en font partie. Le chapitre 12 recense les voies diverses et indirectes que peuvent prendre les traductions : celles du "détour"(Umwegübersetzungen), qui regroupent les traductions de traductions (Übersetzungen aus zweiter Hand, indirect translations, 
traductions au carré), les autotraductions, les rétrotraductions (Rückübersetzungen) qui visent à retrouver l'original à partir d'une version traduite, les différents types de Bearbeitungen, terme générique qui désigne toute sorte d'interventions sur un texte, jusqu'à l'adaptation très libre, sans oublier la trace (fréquente, semble-t-il) de traductions antérieures. L'examen des différents statuts du traducteur est l'objet du chapitre 13, allant d'une pratique romaine liant loisir et honorabilité (otium cum dignitate, suivant la formule cicéronienne), à une activité professionnelle, doublée du cas particulier du traducteur qui est lui-même auteur. La question des niveaux de langues des textes sources est au centre du chapitre 14 : une distinction est à faire entre des nations comme l'Angleterre et la France, dont la centralisation semble avoir facilité l'intégration des niveaux bas dans la " grande littérature " (Schöne Literatur), à la différence de l'Allemagne et de l'Italie où l'existence de dialectes la retarderait ; de toute façon, les traducteurs ont fort à faire quand il s'agit de rendre dialectes, sociolectes, patois, etc.

La seconde partie se termine sur des interrogations, autant que des mises au point, sur deux questions centrales pour mesurer le rôle des traductions dans la vie culturelle internationale. L'une concerne les règles concernant la " propriété intellectuelle ", notion elle-même sujet à débats récurrents depuis le XVIII siècle ; ce domaine complexe est abordé assez rapidement dans le dernier chapitre 16 en partant de la Convention de Berne (1886) et de ses mises à jour successives qui régularisent, difficilement, le marché de la traduction. Le chapitre 15 traite d'une question proprement historiographique : comment les historiens de la langue, d'une part, ceux de la littérature d'autre part, ont-ils tenu compte des traductions dans leurs travaux respectifs ? C'est l'occasion d'un double bilan ; l'évolution de différentes histoires d'une langue montre que ces problèmes, longtemps négligés et traités surtout pour les années de formation, retiennent désormais davantage lattention. En revanche, le bilan des histoires de la littérature paraît plus mitigé : un aperçu des travaux de ce genre parus en Allemagne, en Angleterre et dans les pays de langues romanes montre que la part accordée aux traductions est variable, mais reste assez faible dans l'ensemble. La dernière phrase de ce chapitre - qui aurait peut-être mieux trouvé sa place en conclusion ? - résonne comme un appel : «Il n’y a pas, jusqu’à présent, de consensus parmi les historiens des langues et des littératures sur l'étendue de la place à faire aux traductions dans les histoires d'une langue ou d'une littérature » (463).

La brève Conclusion qui suit rappelle en tout cas que les traductions sont inséparables de l'histoire de la langue, de la littérature et de la culture. Une Bibliographie substantielle, structurée et facile à consulter, comprend une section " fondamentale » comportant elle-même six rubriques et une section "particulière " qui donne d'une part les références des œuvres citées dans l'ouvrage, d'autre part celles des travaux scientifiques utilisés (avec renvois éventuels à la section fondamentale). Cette Bibliographie présente l'avantage, dans sa partie fondamentale, de souvent préciser, en quelques mots, l'intérêt de l'ouvrage mentionné. L'Index, enfin, regroupe environ 300 noms (ceux des auteurs et traducteurs cités dans l'ouvrage).

Europäische Übersetzungsgeschichte présente un bilan équilibré sur le rôle des échanges littéraires réalisés par les traductions dans les six «grandes » langues du long 
chapitre 9. En s'aventurant un peu au-delà de ces langues, les auteurs ont conscience, à juste titre, d'avoir « saisi quelques détails intéressants qui n’ont été jusqu'ici qu’à peine mentionnés dans d’autres exposés d'ensemble " (321). Ce faisant, ils offrent une synthèse malgré tout limitée de l'activité traductive en Europe, car ils sont tributaires de l'état de la documentation et des recherches qui ont, jusqu'à une date récente, privilégié certains domaines linguistiques. Une zone, en particulier, a connu une instabilité linguistique et politique, avec des déplacements de populations, des démembrements et des incorporations dans des ensembles étatiques : de la Bohême à l'Ukraine, de la Finlande à la Bulgarie, on trouve une vingtaine de nations constituées en États plus récemment que dans les pays d'Europe de l'ouest. Dans ces pays aussi dont, pour la plupart, les vestiges linguistiques et historiques sont plus récents, les traductions ont joué un rôle de premier plan.

C'est précisément ce que met en lumière un important ouvrage collectif paru en 2019, soit un an après l'ouvrage d'Albrecht et Plack, Histoire de la traduction littéraire en Europe médiane. Des origines à 1989. Ce travail d'un type nouveau, réalisé par une équipe de 25 contributeurs sous la direction d'Antoine Chalvin, Jean-Léon Muller, Katre Talviste et Marie Vrinat-Nikolov, repose sur l'hypothèse que l'Europe "médiane ", constituée par 16 zones linguistiques situées entre les régions de langue germanique et celle de langue russe, constitue une " aire traductionnelle cohérente " (7). La cohérence de cette aire est forte : rôle fondateur de la Bible, constitution tardive des États nationaux actuels, position périphérique durable par rapport aux courants culturels de l'Europe occidentale, impact politique et culturel d'une puissance extérieure, l'Union soviétique, dans la seconde moitié du $\mathrm{XX}^{\mathrm{e}}$ siècle. Une de ses principales caractéristiques est que la traduction a joué un rôle capital dans l'évolution de ces pays (7-8). La notion d'Europe médiane elle-même est une notion construite récemment - elle vient s'ajouter à celles, plus anciennes et également discutées, d'Europe centrale ou de Mitteleuropa - qui constitue un espace multilingue dont les frontières extérieures et intérieures n'ont cessé d'être mouvantes : l'avant-dernier avatar a été un profond remaniement des frontières, suivi de l'enfermement du « rideau de fer » qui a séparé une partie de ces pays de ceux de l'Europe de l'ouest. Les événements postérieurs à l'année 1989 témoignent d'ailleurs que rien n'est figé dans cette Europe médiane, ni d'ailleurs dans l'ensemble du continent européen.

Europäische Übersetzungsgeschichte et Histoire de la traduction littéraire en Europe médiane se complètent, tout en suivant des options méthodologiques différentes, dues à la différence des champs de recherche. Le premier, œuvre de deux romanistes chevronnés, traite de quelques littératures bien ancrées dans la partie européenne du canon de la Weltliteratur : il suit la ligne de crête des littératures qui ont joué un rôle central, ou semi-central, dans l'histoire de la littérature européenne. Le second, ouvrage collectif d'un groupe international, est le résultat d'une longue enquête sur des littératures qui n'ont pris qu'assez récemment leur essor en s'insérant, parfois à marches forcées, dans les phénomènes de modernité à la fin du XIX siècle, voire au début du $\mathrm{XX}^{\mathrm{e}}$ : encore peu intégrées au canon européen au début du XXI ${ }^{\mathrm{e}}$, ces littératures peuvent effectivement être qualifiées de périphériques. À eux deux, toutefois, ces volumes couvrent l'ensemble de l'espace européen. Celui-ci est hétérogène 
à bien des égards : son histoire est largement constituée par des conflits et des guerres meurtrières, ses limites orientales restent floues, et il n'a d'unité ni climatique, ni linguistique, ni religieuse, ni politique ; les populations qui s'y sont peu à peu fixées n'ont d'ailleurs pris que tardivement conscience qu'elles pouvaient appartenir à un même ensemble.

Les deux ouvrages prennent eux-mêmes place dans une suite de travaux consacrés à des histoires partielles de la traduction - ou des traductions - en Europe parues depuis le début du XXI ${ }^{e}$ siècle (entre autres Lafarga et Pegenaute 2004 ; France et Gillespie 2005-2010 ; Chevrel et Masson 2012-2019 ; Bednárová 2013). Leur confrontation permet d'avoir une approche de quelques-uns des problèmes généraux posés par de telles entreprises.

Un premier problème est celui de l'extension du terme traduction lui-même. Albrecht et Plack affirment, dans leurs remarques préalables $(2018,15)$ : «À côté de ce qu'on appelle les Belles Lettres il faut aussi prendre en compte les textes religieux, philosophiques » et, ajoutent-ils "professionnels " (fachliche). Leur ouvrage, toutefois, s'intéresse presque exclusivement aux Belles Lettres - surtout la prose fictionnelle, quelques poètes ou hommes de théâtre - et à la Bible (ch. 6), et cite simplement quelques philosophes (Kant, Kierkegaard, Nietzsche), sans aller au-delà. En cela ils suivent une position bien établie chez les historiens de la traduction : privilégier les œuvres proprement littéraires - l'adjectif littéraire (ou ses équivalents sémantiques) figure dans plusieurs titres. Outre l'Histoire de la traduction littéraire en Europe médiane, citée plus haut, on a par exemple The Oxford History of Literary Translation in English (France et Gillespie 2005-2010), ou Dejiny umeleckého prekladu na Slovensku (Histoire de la traduction littéraire en Slovaquie I. Du sacré vers le profane, Bednárová 2013) ; il est vrai que dans ces deux ouvrages il est précisé qu'à l'occasion le corpus traité peut s'étendre au-delà de la littérature au sens strict : les traductions de la Bible font partie du corpus traité. Quand le titre n'inclut pas cet adjectif, il est, en fait, quasi-implicite : suivant la même tradition qu'Albrecht et Plack, Francisco Lafarga et Luis Pegenaute renvoient presque exclusivement à des références littéraires dans leur Historia de la traducción en España (2004). Dans Histoire des traductions en langue française (Chevrel et Masson 2012-2019), en revanche, on trouve une tentative pour prendre explicitement en considération l'ensemble des domaines de la "vie de l'esprit » au sens large : philosophie, religion, histoire, droit, sciences. En tout état de cause, on doit considérer qu'il y a là matière à débat : dans la constitution d'une culture européenne, quels sont les domaines à prendre à retenir ?

Un second problème est d'ordre proprement historiographique : dans la mesure où toute histoire implique un effort de périodisation, est-il réellement possible d'envisager une histoire "paneuropéenne » de la traduction, quand il est constant que les pays en cause ont évolué à des rythmes différents et avec des rôles différents? Une réponse claire ne peut pas être préjugée, étant donné les multiples avatars d'un espace européen nullement homogène qui est constitué de zones - pour prendre un terme neutre - qui sont loin d'avoir connu des évolutions semblables. L'exemple de l'Histoire de la traduction littéraire en Europe médiane met en évidence les difficultés. Les éditeurs avaient à traiter seize langues, dont les évolutions ne sont pas synchrones. Ils 
ont choisi d'organiser leur volume en quatre grandes parties chronologiques, qui sont autant d' «étapes dans l'évolution du paradigme traductionnel » : en fait les deux premières parties, qui s'étendent jusqu'à l'orée du $\mathrm{XX}^{\mathrm{e}}$ siècle, ne vont pas sans quelques chevauchements, comme ils le reconnaissent d'ailleurs, mais ils s'efforcent, dans les deux dernières (de la «modernité littéraire » à l'époque du totalitarisme), d'établir des évolutions parallèles entre groupes de langues (centre, sud-est, ensemble baltique). Les volumes de The Oxford History of Literary Translation in English forment eux aussi une suite chronologique de périodes délimitées parfois par un repère historique précis (par exemple : 1650 pour le début du tome 3), parfois par une césure apparemment arbitraire (1900 pour la fin du tome 4) ; mais, à l'intérieur des volumes, les grands chapitres sont constitués tantôt par une succession des langues sources traduites, tantôt par des chapitres thématiques regroupant, par exemple, la «popular culture » ou l'ensemble « Philosophy, History and Travel Writing ». Historia de la traducción en España reprend simplement, dans le domaine du castillan, la périodisation traditionnelle de la littérature espagnole (ce qui confirme que la littérature est le fil conducteur de l'ouvrage), tandis qu'Histoire des traductions en langue française, qui prend son point de départ avec l'invention de l'imprimerie pour s'achever au tournant des $\mathrm{XX}^{\mathrm{e}}-\mathrm{XXI}^{\mathrm{e}}$ siècles prend de grands repères à l'échelle de l'histoire européenne (comme 1815, 1914/1918), et s'efforce de proposer des périodisations différentes suivant les grands domaines traités dans les chapitres. Ces choix différents se retrouvent dans ceux qu'Albrecht et Plack avaient suivis successivement : une perspective globale de l'Antiquité au Moyen Âge puis de la Renaissance au Romantisme, entrecoupée par trois chapitres thématiques (dont celui consacré à la Bible), avant d'en venir aux importants développements du chapitre 9, dans lequel l'histoire des traductions est alors présentée dans six espaces linguistiques, dont chacun a sa périodisation propre.

L'ensemble des travaux mentionnés contribue incontestablement à faire mieux comprendre la place qu'ont occupée les traductions dans l'histoire de certaines parties de l'Europe. D'autres projets sont actuellement en cours de réalisation. ${ }^{1}$ Une question nouvelle peut alors se poser : est-il possible d'aller au-delà et de penser à une histoire " paneuropéenne » de la traduction, c'est-à-dire à une histoire qui prendrait comme objet la circulation des traductions sur la totalité du territoire européen ? Les deux grands problèmes évoqués prendraient encore davantage d'acuité, d'autant que la documentation à traiter, de plus en plus aisément accessible grâce aux technologies numériques, serait considérable. Mais, instruits par les expériences déjà menées à bien, le temps semble venu pour les chercheurs de s'intéresser aussi aux trajets des trajets des textes traduits, en analysant les formes sous lesquelles ils ont circulé (parfois à partir de traductions dans d'autres langues), afin de mettre en évidence les circuits de ces échanges, dans lesquels toutes les langues d'Europe ont leur place.

\section{NOTES}

1 Par exemple Istoria traducerilor în limba română (Histoire des traductions en langue roumaine), projet dirigé par Muguraș Constantinescu et Rodica-Mărioara Nagy, université Ștefan cel Mare (Suceava, Roumanie). 


\title{
BIBLIOGRAPHIE
}

Albrecht, Jörn et Iris Plack. 2018. Europäische Übersetzungsgeschichte. Tübingen : Narr Francke Attempto Verlag.

Bednárová, Katarína. 2013. Dejiny umeleckého prekladu na Slovensku I. Od sakrálneho k profánnemu. Bratislava : Veda, vydavatelstvo SAV.

Chalvin, Antoine, Jean-Léon Muller, Katre Talviste et Marie Vrinat-Nikolov, dir. 2019. Histoire de la traduction littéraire en Europe médiane des origines à 1989. Rennes : Presses Universitaires de Rennes.

Chevrel, Yves et Jean-Yves Masson, dir. 2012-2019. Histoire des traductions en langues française. Volume 1-4. Lagrasse : Editions Verdier.

France, Peter et Stuart Gillespie, dir. 2005-2010. The Oxford History of Literary Translation in English. Volume 1-4. Oxford: Oxford University Press.

Hoof, Henri Van. 1991. Histoire de la traduction en Occident. Bruxelles : Duculot.

Lafarga, Francisco et Luis Pegenaute, dir. 2004. Historia de la traducción en España. Salamanca : Editorial Ambos Mundos.

\section{About a recent European history of translation}

History of translation(s). Peripherical languages. Cultural turn.

Under what conditions is it possible to elaborate a history of translation(s) in the European area? Reading Jörn Albrecht's and Iris Plack's Europäische Übersetzungsgeschichte [European Translation History] and some other recent publications, some questions arise, such as: how can one take into account the part of translations in the so-called peripheric languages? Is literature the main (or only) way to investigate and to study the role of translation(s), or would it be better to extend to other fields (religion, history, science)? Can translations lead to cultural turns?

\author{
Yves Chevrel \\ Professeur émérite \\ Sorbonne Université \\ 3 allée de Beauvais \\ F - 35830 Betton \\ France \\ yves.chevrel35830@gmail.com
}

